\title{
Author Index of Volume 216 Issue 1
}

Adam. W. 123

Ahtgren. M.. 55

Alte da Veiga. L.. 129

Amann. P.. 17

Artico. M.. 57

Baldé. L.. 59

Becker. P.. 31. 35

Bleckmann, P.. 63.65.67

Boche. G.. 143

Böcker. M.. 19

Bohatý, L.. 33

Böhmer, N.. 19

Bonnetot. B., 115

Bosold, F.. 143

Boy, I., 7.9.11,13

Bringmann, G., 119

Carrillo-Cabrera, W., 42, 43

Cérésiat, M., 51, 53

Chammache. M., 101

Christl, M., 121

Comu, D.. 115

Crisma, M., 103

Ezank, M., 39

Dąbrowski, J., 126

Dadachov, M. S., 141

De Lucchi, O., 103, 117

Declercq. J.-P. 51, 53

Deiseroth, H. J., 25, 27

Dey, B. K., 71

Dey, L., 7I

Drexler, H.-J., 165, 169

Duddeck, H., 151

Eisenmann, B., 36

Endress, H., 119

Eriksson, L., 145

Essassi, E. M., 101

Ettorre, A., 57

Evans, C. C., 61

Fabris, F., 117

Fehring, V., 157

Flemström, A., 41

Fornasini, M. L., 21, 23, 24

Frey, W., 91, 93, 95, 97

Fröhlich, R., 31

Fur, Y. Le, 61

Gerlitzki, N., 19

Gesing, Th. M., 37

Gieck, C., 36

Glavcheva, Z., 67

Gloe, K., 151

Göbel, H., 29

Grin, Yu., 42

Groetsch, S.. 121

Haikarainen, A., 147

Hämäläinen, R., 132

Hansing, J., 17

Harms, K., 143

Hazari, S. K. S., 71

Hegetschweiler, K., 105, 108, 111,113

Heinz, U., 113

Held, P., 33. 35
Heller. D.. 157, 161, 165, 169

Henkel, S., 89

Hesse. K.-F.. 39

Hilgers. P.. 91, 93, 95

Horiuchi. A., 73

Horn, E., 71. 73. 75. 77. 80. 83

Huang. Y.-X.. 15

Huch, V., 105

Hussain, A.. 37

Irngartinger, $H_{\text {., }} 126$

Jäger, V.. 89, $91,93,95$

Jeitschko, W., 1, 3

Jouan, M., 59

Julien, R., 59

Kadyrov, R., 157

Kantlehner, W., 97

Kempe, R., 153, 157, 161, 165

Kleb, D. Chr., 63, 65, 67

Kleban, M., 89, 91

Klinga, M., 132

Kniep, R., 5, 7, 9, 11, 13, 15

Kolev, T,, 63, 65, 67

Kong, Z.-P., 137

Kristiansson, O., 86

Kuppert, D., 111

Kurosawa, K., 77

La Colla, P., 57

Laitinen, R. S., 55

Lazzari, D., 103

Lecocq, S., 115

Leithe-Jasper, A., 45

Li, S.-L., 83

Librera, C. P., 123

Lidin, S., 41

Lin, J.-L., 135, 137, 139

Mao, S.-Y., 15

Marchand-Brynaert, J., 51, 53

Marsch, M., 143

Massa, S., 57

Masse, R., 61

Matos Beja, A., 129

Mazzone, D., 21

Merlo, F., 23, 24

Meyer, G., 18, 19, 20

Mi, J.-X., 15

Michieletto, I., 117

Miele, P., 115

Möller, A., 17

Mormann, Th. J., 1, 3

Mutikainen, I., 147

Nabeshima, T., 80

Nakahodo, T., 77

Nemcsics, Á., 39

Niewa, R., 5

Nishida, D., 80

Oeser, T., 126

Oilunkaniemi, R., 55

Onai, S., 75

Oppermann, H., 29

Paixāo, J. A., 129

Pajunen, A., 147
Pani. M. 23, 24

Peters. E.-M.. 117. 119. 121, 123

Peters, K., 42, 43, 117, 119, 121, 123

Pierrot. M.. 10

Pirelahi. H., 47

Possenti, M.. 103

Preut, H., 63. 65. 67

Rademacher, O., 29.151

Rahmani, H., 47

Ramos Silva, M., 129

Rebanda, N. G. G. L.. 129

Riani, P.., 21

Rocha Gonsalves, A. M. d'A., 129

Roos, M., 18

Rößler, U., 36

Roth, C., 111

Roy, T. G., 71

Ruck, M., 29

Rüscher, C. H., 37

Sander, J., 105, 108, 111, 113

Sato, A., 45

Schäfer, G., 11, 13

Scherr, O., 97

Schlirf, J., 25, 27

Schnering, H. G. von, 42, 43

Schürmann, M., 63, 65, 67

Silvestre, J.-P., 59

Silvestri, R., 57

Sipilä, J., 147

Sobral, A. J. F. N., 129

Somer, M., 42, 43

Soverini, M., 103

Spannenberg, A., 153, 157, 161, 165, 169

Sun, J., 135

Tadjarodi, A., 47

Taeb, A., 47

Tamura, H., 77

Tanaka, T., 45

Tang, L.-Q., 141

Tasler, S., 119

Tiekink, E. R. T., 69, 71

Tinant, B., 51, 53

Toury, B., 115

Turpeinen, U., 132

Uzoukwu, B. A., 151

Veith, M., 105

Wadsten, T., 145

Wei, Z.-B., 15

Werner, P.-E., 145

Winter, M., 108

Yancheva, D., 63, 65, 67

Yao, Q., 99

Zanicchi, G., 21

Zhang, M., 83

Zhao, J.-T., 15

Zheng, C., 99

Zheng, Y.-Q., 135, 137, 139

Zhou, L.-X., 137

Ziegler, G., 97

Zou, X.-D., 141 